\title{
Stressed waveguides with tubular depressed-cladding inscribed in phosphate glasses by femtosecond hollow laser beams
}

\author{
Xuewen Long, ${ }^{1,2}$ Jing Bai, ${ }^{1,2}$ Wei Zhao, ${ }^{1}$ Razvan Stoian, ${ }^{3}$ Rongqing Hui, ${ }^{4}$ and Guanghua Cheng ${ }^{1, *}$ \\ ${ }^{1}$ State Key Laboratory of Transient Optics and Photonics, Xi'an Institute of Optics and Precision Mechanics, \\ Chinese Academy of Sciences, Xi'an 710119, China \\ ${ }^{2}$ Graduate University of Chinese Academy of Sciences, Beijing 100049, China \\ ${ }^{3}$ Laboratoire Hubert Curien, UMR 5516 CNRS, Université de Lyon, Université Jean Monnet, 42000 Saint Etienne, France \\ ${ }^{4}$ Department of Electrical Engineering and Computer Science, University of Kansas, Lawrence, Kansas 66044, USA \\ ${ }^{*}$ Corresponding author: gcheng@opt.ac.cn
}

Received May 8, 2012; revised June 20, 2012; accepted June 27, 2012; posted June 28, 2012 (Doc. ID 168104); published July 23, 2012

\begin{abstract}
We report on the single-step fabrication of stressed optical waveguides with tubular depressed-refractive-index cladding in phosphate glasses by the use of focused femtosecond hollow laser beams. Tubelike low index regions appear under direct exposure due to material rarefaction following expansion. Strained compacted zones emerged in domains neighboring the tubular track of lower refractive index, and waveguiding occurs mainly within the tube core fabricated by the engineered femtosecond laser beam. The refractive index profile of the optical waveguide was reconstructed from the measured transmitted near-field intensity. (c) 2012 Optical Society of America

OCIS codes: $140.3390,230.7370$.
\end{abstract}

Femtosecond (fs) laser waveguide writing in bulk transparent materials has attracted a considerable interest for integrated three-dimensional (3D) photonics. This is mainly due to the potential of optical processing with fewer technological steps and improved geometrical flexibility in comparison with alternative waveguide fabrication techniques [1]. In several glasses of optical interest (e.g., fused silica), when fs laser pulses are focused inside the dielectric material at mild energetic doses, a positive change in the refractive index is produced within the laser photowritten trace. In such a way, optical waveguides could be generated directly along single tracks. However, in some materials including a large range of glasses, crystals, and polymers, ultrafast laser exposure produces merely a negative refractive index variation due to matter rarefaction or volume damage in the laser induced tracks [2-5]. Therefore, light guidance within a single track becomes impossible, triggering a search of alternative ways to overcome the situation. In fact, subsequent to irradiation and material expansion, strained regions always emerge in the vicinity of these tracks. Here, following compaction, the refractive index may be higher in comparison with the substrate, allowing partial waveguiding. This suggests already techniques of waveguide writing using the collateral stress-induced positive refractive index variation for guiding light []․ Using this concept, pair line structure consisting of two parallel tracks on each side of the unexposed central domain was successfully demonstrated to confine light [글 $\underline{10}$. More complicated closed-form structures such as tubular designs consisting of a number of parallel tracks have also been demonstrated to confine light and to generate laser effects in $\mathrm{ZrF}_{4}-\mathrm{BaF}_{2}-\mathrm{LaF}_{3}-\mathrm{AlF}_{3}-\mathrm{NaF}$ (ZBLAN) fluoride glass [11]. In those structures, multiple scans are necessary to form a tubular laser trace in transverse scanning mode. A more direct approach can be anticipated, where a hollow laser beam may be used with scanning along the laser propagation axis to fabricate waveguides with excellent symmetry in a single-step scanning process.

In this Letter, we report on the fast single-step writing of stressed waveguides with tubular depressedrefractive-index cladding in phosphate glasses by the use of focused fs hollow beams. This procedure takes advantage of laser-induced low-index generation under direct exposure of the engineered light and side accumulation of compaction. Employing this novel method, we can increase the processing efficiency and eliminate mutual side effects of damage tracks induced by subsequent laser pulses during the multiscan writing process arising from their close proximity. In addition, a hollow laser beam is beneficial to decrease spherical aberration due to refraction at air-dielectric interfaces [12].

In fact, a focused hollow beam can be directly generated by focusing higher-order nondiffracting Bessel beams. The proposed higher-order Bessel function beams have a zero axial irradiance, with the light being ringlike collimated around the axis. Their intensity profiles are invariant during propagation over extended distances. Nondiffracting zero-order Bessel beams were successfully demonstrated for material processing without sample translation [13]. The truncated higher-order Bessel beams proposed here can be created with a hologram employing a phase shift defined such as [14]

$$
T_{n}(r, \theta)=\exp (i n \theta) \exp \left(-i \frac{2 \pi}{r_{0}} r\right),
$$

where $r$ and $\theta$ are transverse polar coordinates, $r_{0}$ is an adjustable scale factor, and $n$ is an integer. The electric field of the $n$ th-order truncated Bessel beam formed after the plane of the hologram is approximately given by

$$
E_{n}(r, \theta, z)=J_{n}\left(\frac{2 \pi}{r_{0}} r\right) \exp (i n \theta) \exp (i \beta z)
$$


where $J_{n}(\cdot)$ is the $n$ th-order Bessel function of the first kind and $\beta^{2}=k^{2}-\left(2 \pi / r_{0}\right)^{2}$. Using the identity $F\left\{g(r) e^{i n \theta}\right\}=(-i)^{n} e^{i n \theta} H_{n}\{g(r)\}$, where $F\{\cdot\}$ and $H_{n}\{\cdot\}$ are the Fourier transform and the Hankel transform of order $n$, respectively [15], the Fourier transform of Eq. (2) is directly proportional to $\delta\left(r / \lambda f-1 / r_{0}\right)$, where $\delta(\cdot)$ is the Dirac delta function and $f$ is the focal length of the lens. Thus, a ring-shaped intensity profile can be generated at the focal plane of the lens by focusing higher-order Bessel beams. The size of the ring at the focal plane can be tuned by changing the scale factor $r_{0}$. As is well known, multiphoton absorption confines laser interactions to the focal volume and reduces collateral damage in the surrounding material. Therefore, a tubular structure can be fabricated by scanning the foci of the engineered fs laser beam parallel to the direction of laser propagation.

The experimental setup used for generating focused fs hollow beams and fabricating stressed optical waveguides with tubular depressed-refractive-index cladding is schematically shown in Fig. 1. Fs laser pulses operating at a repetition rate of $1 \mathrm{kHz}$ and pulse duration of $120 \mathrm{fs}$ with a center wavelength of $800 \mathrm{~nm}$ are generated by a Ti: sapphire CPA laser system (Spitfire, Spectra Physics). These pulses are reflected on a computer-controlled and electrically addressed spatial light modulator (SLM) (HEO1080P, HOLOEYE Photonics AG) for beam shaping. The SLM matrix has $1920 \times 1080$ pixels, and its active area is $15.36 \times 8.64 \mathrm{~mm}^{2}$. The spatial phase distributions of the laser beam were modulated by the calibrated SLM, relying on the local refractive index variations of the liquid crystal map as the function of the driving voltage. A higher-order Bessel beam with $n=10$ and $r_{0}=0.8 \mathrm{~mm}$ was generated after the SLM, which modulated the spatial phase of the incident wave according to Eq. (1). The focusing lens is placed in the Bessel beam collimation zone. Subsequently, a ring-shaped intensity profile can be created at the back focal plane of the focusing lens (Aspheric lens, focal length $f=8 \mathrm{~mm}$, working distance $5.92 \mathrm{~mm}$, C240TME-B, Thorlabs). The phase mask loaded on the SLM and the produced ring-shaped beam waist profile in free-space are shown in Fig. 2. In order to obtain a higher beam quality, we inserted in addition a phase term of a tilted plane wave to spatially separate and filter away the zero-order of diffraction, which originates from the SLM itself.

The ring-shaped intensity profile generated around the focal region was used to inscribe the tubular structures

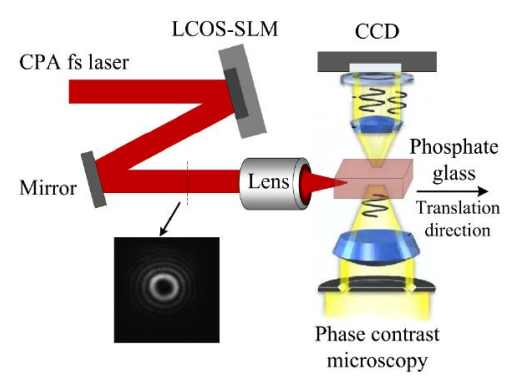

Fig. 1. (Color online) Schematic representation of the experimental setup. The inset represents the spatial profile of the higher-order nondiffracting Bessel beam located between the spatial light modulator (SLM) and the focusing lens.

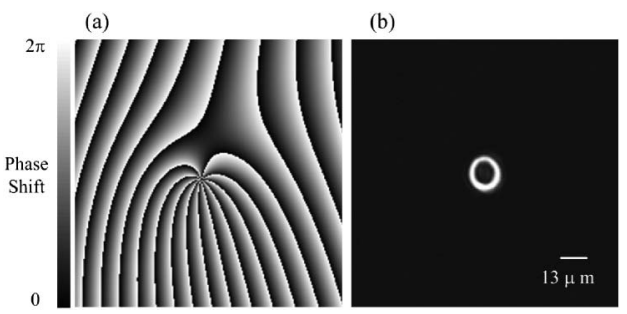

Fig. 2. (a) Phase mask employed for producing a Bessel beam with $n=10$ and $r_{0}=0.8 \mathrm{~mm}$. (b) Transverse intensity distribution at the focus of lens in air. The scale bar is $13 \mu \mathrm{m}$.

of material modification in a $4 \times 10 \times 30 \mathrm{~mm}^{3}$ polished phosphate glass parallelepiped using a longitudinal scheme. The sample was mounted on computer controlled high precision positioning stages (Physik Instrumente) and translated parallel to and towards the laser beam propagation direction at a rate of $5 \mu \mathrm{m} / \mathrm{s}$. For the fabrication of a high quality structure, the pulse energy is required not to exceed the threshold, which may otherwise result in beam collapse. In the experiment, the ontarget pulse energy was set to $6 \mu \mathrm{J}$, and pulse duration was set to $340 \mathrm{fs}$. An Olympus BX51 positive phase contrast microscope (PCM) was employed to image the interaction region in a side-view geometry, showing relative variations of the refractive index translated into an intensity map. The image was recorded with a chargecoupled device (CCD) camera. Figure 3(a) depicts the side-view PCM image of the tubular structure. Two lines represent the side wall of the tube. The PCM image shows that the tubular track experienced a negative refractive index variation with respect to the pristine glass matrix [note white color in Fig. 3(a) indicative of negative index changes in positive $\mathrm{PCM}]$. The negative index change in exposed regions can most probably be related to the lower density as discussed below. Complementing the PCM information, the optical transmission microscope image of the exit plane of the obtained tubular structure is shown in Fig. 3(b), indicating the confinement of the laser modification. The diameter of the tubular track is $12 \mu \mathrm{m}$. In our exposure conditions, during photoinscription, the deposition of the fs laser energy leads to a decrease of the local density and thus of the refractive index in the tubular track due to thermal expansion at the point of exposure of the laser beam [16]. The thermally driven expansion, even for low repetition rates, causes compaction in the surrounding
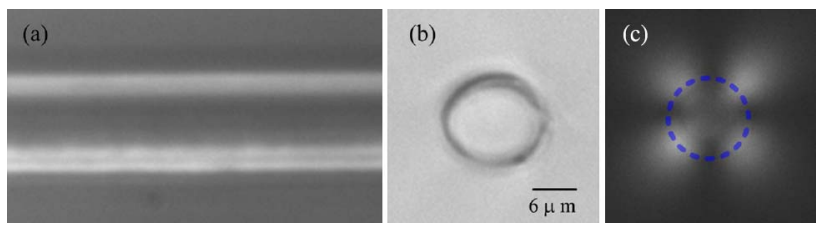

Fig. 3. (Color online) (a) Phase contrast microscope (PCM) image of the waveguide with tubular depressed-refractive-index cladding (side view). Note the negative index change (white color) in the lateral zones. (b) Optical transmission micrograph of the obtained tubular structure (end view). (c) Microscope image of the tubular track between crossed polarizers. The dashed blue circle marks the position of the track. The field of view is the same. The scale bar is $6 \mu \mathrm{m}$. 

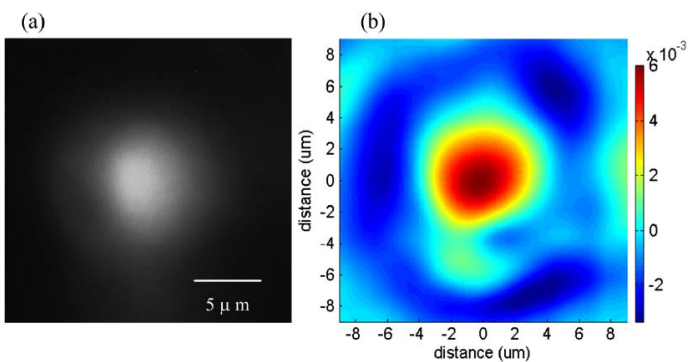

Fig. 4. (Color online) (a) Near-field mode of the tubular waveguide injected by $800 \mathrm{~nm} \mathrm{CW}$ laser radiation. (b) Calculated relative refractive index distribution of the waveguide corresponding to the near-field mode presented in (a).

regions, which is responsible for an increase of the refractive index on axis, within the laser unexposed core. To check eventual stress-induced polarization effects, in Fig. 3(c), the tubular track under cross-polarization microscopy is shown. The polarizer was positioned underneath the sample stage with its pass axis fixed in the east-west direction and the analyzer aligned with a pass axis oriented north-south. The dashed blue circle marks the position of the track. Four bright petals pattern occurs and the orientation of the four petals stays unchanged when the sample is rotated relative to the polarizer due to a radial distribution of stress. The angles between the orientation of maximum brightness and the pass direction of the polarizer are $45,135,225,315^{\circ}$, respectively \{see Eq. (3) in [5]\}. Given the symmetry of compression, birefringence cannot take place in the center of the core [under cross-polarization microscopy, extinction takes place in the center of the core shown in Fig. 3(c)]. The increase of refractive index within the core of the tube due to stress-induced densification and depressed-refractive-index cladding supports efficient light propagation. The radial distribution of constraints leading to core densification does not induce polarization dependent optical guiding effects for the injected light. An attenuated $800 \mathrm{~nm} \mathrm{CW}$ laser radiation was coupled to one end facet of the waveguide by the aspheric lens mentioned above. The output emerging from the other end of the waveguide was imaged with a $20 \times$ microscope objective (N.A. 0.42) and recorded with a CCD camera. The near-field mode is about $13 \mu \mathrm{m}$ in diameter (at $1 / e^{2}$ level) with a perfect single mode intensity profile, as shown in Fig. 4(a). A finite differences method for the reconstruction of refractive index profiles from near-field mode has been employed here to obtain the relative refractive index profile of the stressed-induced optical waveguide [17]. The maximum refractive index change is 0.006, as shown in Fig. 4(b), sufficient for single mode guiding. We recall that the size of the core can be also changed by the use of the phase mask with different parameter $r_{0}$, leading to increased flexibility in the modal properties of the guide.
In summary, we introduced a method of single-step fabrication of stressed-induced optical waveguide with tubular depressed-refractive-index cladding in phosphate glasses by the use of focused fs hollow laser beams. Flexibility in guide design can be obtained by the parameters of the engineered beams. The refractive index profile of optical waveguide was also reconstructed from the measured transmitted near-field mode profile.

The authors acknowledge the support given by the West Light Foundation of The Chinese Academy of Sciences (No. 0729591213), the Innovative Research International Partnership Project of The Chinese Academy of Sciences, and the French Agence Nationale de la Recherche.

\section{References}

1. K. M. Davis, K. Miura, N. Sugimoto, and K. Hirao, Opt. Lett. 21, 1729 (1996).

2. J. W. Chan, T. R. Huser, S. H. Risbud, J. S. Hayden, and D. M. Krol, Appl. Phys. Lett. 82, 2371 (2003).

3. J. Siegel, J. M. Fernández-Navarro, A. García-Navarro, V. Diez-Blanco, O. Sanz, J. Solis, F. Vega, and J. Armengol, Appl. Phys. Lett. 86, 121109 (2005).

4. A. Zoubir, C. Lopez, M. Richardson, and K. Richardson, Opt. Lett. 29, 1840 (2004).

5. J. Morikawa, A. Orie, T. Hashimoto, and S. Juodkazis, Opt. Express 18, 8300 (2010).

6. T. Gorelik, M. Will, S. Nolte, A. Tuennermann, and U. Glatzel, Appl. Phys. A 76, 309 (2003).

7. W. F. Silva, C. Jacinto, A. Benayas, J. R. Vazquez de Aldana, G. A. Torchia, F. Chen, Y. Tan, and D. Jaque, Opt. Lett. 35, 916 (2010).

8. A. Benayas, W. F. Silva, C. Jacinto, E. Cantelar, J. Lamela, F. Jaque, J. R. Vázquez de Aldana, G. A. Torchia, L. Roso, A. A. Kaminskii, and D. Jaque, Opt. Lett. 35, 330 (2010).

9. J. Siebenmorgen, T. Calmano, K. Petermann, and G. Huber, Opt. Express 18, 16035 (2010).

10. G. A. Torchia, A. Rodenas, A. Benayas, E. Cantelar, L. Roso, and D. Jaque, Appl. Phys. Lett. 92, 111103 (2008).

11. D. G. Lancaster, S. Gross, H. Ebendorff-Heidepriem, K. Kuan, T. M. Monro, M. Ams, A. Fuerbach, and M. J. Withford, Opt. Lett. 36, 1587 (2011).

12. C. Mauclair, A. Mermillod-Blondin, N. Huot, E. Audouard, and R. Stoian, Opt. Express 16, 5481 (2008).

13. M. K. Bhuyan, F. Courvoisier, P. A. Lacourt, M. Jacquot, R. Salut, L. Furfaro, and J. M. Dudley, Appl. Phys. Lett. 97, 081102 (2010).

14. N. Chattrapiban, E. A. Rogers, D. Cofield, W. T. Hill, and R. Roy, Opt. Lett. 28, 2183 (2003).

15. J. W. Goodman, Introduction to Fourier Optics, 2nd ed. (McGraw-Hill, 1996).

16. A. Mermillod-Blondin, I. M. Burakov, Yu. P. Meshcheryakov, N. M. Bulgakova, E. Audouard, A. Rosenfeld, A. Husakou, I. V. Hertel, and R. Stoian, Phys. Rev. B 77, 104205 (2008).

17. F. Caccavale, F. Segato, I. Mansour, and M. Gianesin, J. Lightwave Technol. 16, 1348 (1998). 